\title{
Urinary Biomarkers may Complement the Cleveland Score for Prediction of Adverse Kidney Events After Cardiac Surgery: A Pilot Study
}

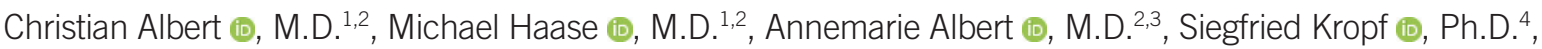
Rinaldo Bellomo (iD, M.D. ${ }^{5}$, Sabine Westphal 무, M.D. ${ }^{6}$, Mark Westerman, Ph.D. ${ }^{7 \dagger}$, Rüdiger Christian Braun-Dullaeus $\mathbb{1 0}$, M.D. ${ }^{8}$, and Anja Haase-Fielitz (i), Pharm.D. ${ }^{9,10}$

${ }^{1}$ Faculty of Medicine, Otto-von-Guericke University Magdeburg, Magdeburg, Germany; '2Diaverum Renal Services, MVZ Potsdam, Potsdam, Germany; ${ }^{3}$ Department of Nephrology and Endocrinology, Klinikum Ernst von Bergmann, Potsdam, Germany; ${ }^{4}$ Institute for Biometrics and Medical Informatics, Ottovon-Guericke University Magdeburg, Magdeburg, Germany; ${ }^{5}$ School of Medicine, University of Melbourne, Melbourne, Australia; ${ }^{6}$ Institute of Laboratory Medicine, Hospital Dessau, Dessau, Germany; ${ }^{7}$ Intrinsic LifeSciences, La Jolla, CA, USA; ${ }^{8}$ Department of Internal Medicine, University Clinic for Cardiology and Angiology, Otto-von-Guericke University Magdeburg, Magdeburg, Germany; ${ }^{9}$ Department of Cardiology, Immanuel Diakonie Bernau, Heart Center Brandenburg, Brandenburg Medical School Theodor Fontane (MHB), Germany; ${ }^{10}$ Institute of Social Medicine and Health Economics, Otto-von-Guericke University Magdeburg, Magdeburg, Germany
\end{abstract}

Background: The ability of urinary biomarkers to complement established clinical risk prediction models for postoperative adverse kidney events is unclear. We assessed the effect of urinary biomarkers linked to suspected pathogenesis of cardiac surgery-induced acute kidney injury (AKI) on the performance of the Cleveland Score, a risk assessment model for postoperative adverse kidney events.

Methods: This pilot study included 100 patients who underwent open-heart surgery. We determined improvements to the Cleveland Score when adding urinary biomarkers measured using clinical laboratory platforms (neutrophil gelatinase-associated lipocalin [NGAL], interleukin-6) and those in the preclinical stage (hepcidin-25, midkine, alpha-1 microglobulin), all sampled immediately post-surgery. The primary endpoint was major adverse kidney events (MAKE), and the secondary endpoint was AKI. We performed ROC curve analysis, assessed baseline model performance (odds ratios [OR], 95\% Cl), and carried out statistical reclassification analyses to assess model improvement.

Results: NGAL (OR [95\% Cl] per 20 concentration-units wherever applicable): (1.07 [1.01-1.14]), Interleukin-6 (1.51 [1.01-2.26]), midkine (1.01 [1.00-1.02]), 1-hepcidin-25 (1.08 [1.00-1.17]), and NGAL/hepcidin-ratio (2.91 [1.30-6.49]) were independent predictors of MAKE and AKI (1.38 [1.03-1.85], 1.08 [1.01-1.15], 1.01 [1.00-1.02], 1.09 [1.01-1.18], and 3.45 [1.54-7.72]). Category-free net reclassification improvement identified interleukin- 6 as a model-improving biomarker for MAKE and NGAL for AKI. However, only NGAL/hepcidin-25 improved model performance for event- and event-free patients for MAKE and AKI.

Conclusions: NGAL and interleukin- 6 measured immediately post cardiac surgery may complement the Cleveland Score. The combination of biomarkers with hepcidin-25 may further improve diagnostic discrimination.

Key Words: Acute kidney injury, Cleveland Score, Major adverse kidney events, Cardiac surgery, Hepcidin, Interleukin-6, Midkine, Neutrophil gelatinase-associated lipocalin, Reclassification analysis
Received: June 8, 2019

Revision received: July 9, 2019

Accepted: October 8, 2019

\section{Corresponding author:}

Anja Haase-Fielitz, Pharm.D.

Institute of Social Medicine and Health Economics, Otto-von-Guericke University Magdeburg, Leipziger Straße 44,

Magdeburg 39120, Germany

Tel: +49-391-6724324

Fax: +49-391-6724310

E-mail: Anja.Haase-Fielitz@med.ovgu.de

\section{Co-corresponding author:}

Christian Albert, M.D.

Faculty of Medicine, Otto-von-Guericke University Magdeburg, Diaverum Renal Services, MVZ Potsdam, Germany Am Neuen Garten 11, Potsdam 14469,

Germany

Tel: +49-331-280690

Fax: +49-331-2806932

E-mail: Christian.Albert@Diaverum.com

${ }^{\dagger}$ deceased

\section{(i) (2)}

(c) Korean Society for Laboratory Medicine This is an Open Access article distributed under the terms of the Creative Commons Attribution Non-Commercial License (http://creativecommons.org/licenses/by-nc/4.0) which permits unrestricted non-commercial use, distribution, and reproduction in any medium, provided the original work is properly cited. 


\section{INTRODUCTION}

Acute kidney injury (AKI) is a common and serious complication of cardiac surgery with incremental, stage-dependent worsening of prognosis [1]. The most recent clinical practice guidelines for AKI highlight the importance of earliest possible detection of AKI and adjustment of treatment accordingly [2]. In patients undergoing cardiac surgery, treating physicians may consult clinical variable-based risk assessment models to predict postoperative adverse kidney events, especially the widely adopted Cleveland Score [3, 4]. However, additional pathophysiological information derived from kidney biomarker analysis and clinical information are needed, as risk assessment based solely on preoperative information may not be sufficiently accurate. Additionally, an established clinical risk model combining preoperative and intraoperative information on kidney risk/response is not yet available.

Recently, kidney injury biomarkers have been included in AKI definition and risk assessment to complement established renal functional criteria [5]. However, a candidate biomarker should be able to improve a reference model in order to be of diagnostic or prognostic benefit [6]. Oxido-inflammatory stress and iron metabolism are involved in the pathogenesis of cardiac surgeryassociated AKI [1, 7]. Given that, in a single patient, several pathomechanisms may be simultaneously active to cause AKI, it may be reasonable to analyze biomarkers in the urine linked to oxido-inflammatory stress and iron metabolism, such as interleukin-6, neutrophil gelatinase-associated lipocalin (NGAL), hepcidin-25, and alpha-1 microglobulin.

Interleukin-6 is a proinflammatory acute phase response cytokine that was recently found to be elevated in the urine of patients with acute tubular injury [8, 9]. Both NGAL [10] and hepcidin-25 are regulators of tubular iron metabolism [7, 11]. Alpha-1 microglobulin is another member of the lipocalin superfamily involved in heme degradation, and, when found increased in urine may indicate proximal tubular injury [12]. Midkine cannot be filtered through the glomerular basement membranes, pointing to generation through tubular injury induced by ischemia and hypoxia when found in urine [13, 14]. Postoperatively, increased urinary concentrations of hepcidin- 25 were found in non-AKI patients suggesting renal-protective capability $[15,16]$.

Finally, data regarding prediction of MAKE or AKI in conjunction with the Cleveland Score is unavailable. Therefore, we aimed to identify the urinary kidney injury biomarkers or biomarker combination with the best possible additive predictive ability for adverse kidney-related events post-cardiac surgery. We hypothesized that the predictive ability of above-mentioned urinary biomarkers could improve the predictive performance of the Cleveland Score.

\section{METHODS}

\section{Patients and setting}

This exploratory ancillary study of the BIC-Multicenter Study used a cohort of 100 patients who underwent elective openheart surgery with the use of cardio-pulmonary bypass (CPB), enrolled as a control group at the German Heart Center, Berlin, Germany (NCT00672334) from January 2009 through June 2010 (Fig. 1). Full study details have been described previously [17]. This study was approved by the Institutional Review Board of Charité University Medicine Ethics Committee, Berlin, Germany (approval no.: ZS EK 11 654/07), and written informed consent was obtained from each patient. We excluded patients undergoing emergency operations (time between hospital admission to operation <24 hours) or off-pump surgery, patients presenting with advanced chronic kidney disease (serum creati-

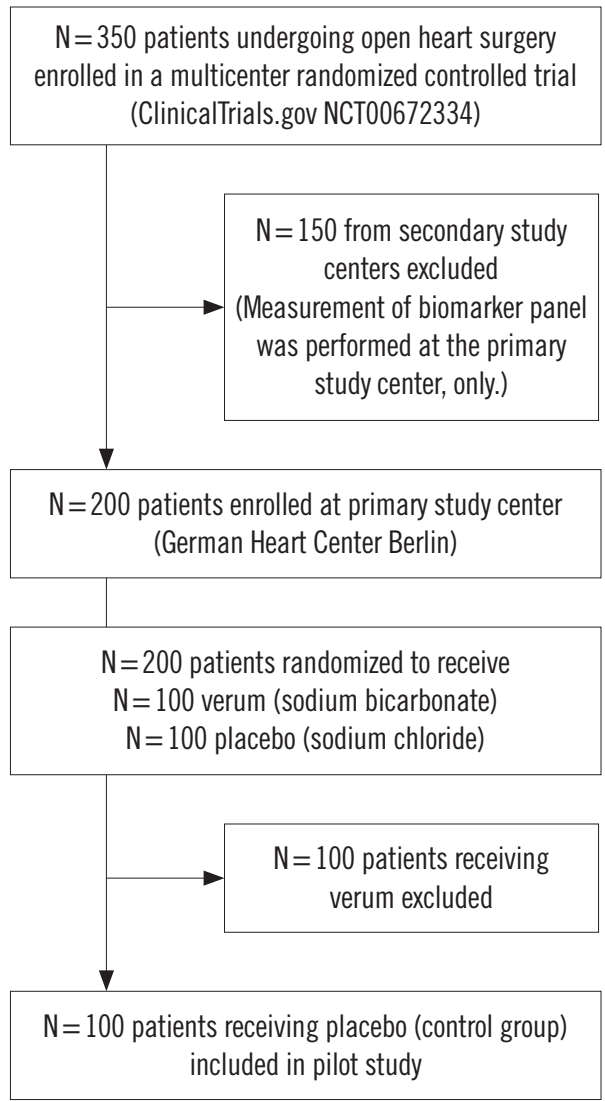

Fig. 1. Patient flow diagram. 
nine $>300 \mu \mathrm{mol} / \mathrm{L}$ ) or kidney transplant, patients $<18$ years, patients on immunosuppression medication, and those enrolled in a conflicting research study. Decisions regarding all diagnostic and therapeutic interventions were performed by the intensive care physicians, independent of this investigation.

\section{Study endpoints}

The primary endpoint was the development of MAKE, including alternatively occurring events of RIFLE-AKI stages Injury or Failure (Risk, Injury, Failure, End-stage renal disease classification [18]), persistent AKI $>48$ hours, acute renal replacement therapy (RRT) initiation, and in-hospital mortality. The secondary endpoint was AKI, defined and classified by severity according to the RIFLE criteria based on increases in postoperative serum creatinine concentration compared with the preoperative baseline concentration, as well as urine output criteria [18]. We chose the RIFLE criteria because they tend to have a higher discriminative value in predicting hospital mortality in cardiac surgery patients than the Acute Kidney Injury Network or Kidney Disease Improving Global Outcome criteria [19, 20].

\section{Biomarker sampling and measurement}

We obtained urine at 6 hours (referred to as 'intensive care unit [ICU] admission') after commencement of CPB. Sampling was performed as previously described [17]. NGAL concentration ( $\mathrm{ng} / \mathrm{mL}$ ) was measured using an ARCHITECT Analyzer (Abbott Diagnostics, Abbott Park, IL, USA). Interleukin-6 (pg/mL) and alpha-1 microglobulin $(\mathrm{ng} / \mathrm{mL}$ ) concentrations were determined using the Cobas e/c411 Immunoassay Analyzer Platform (Roche Diagnostics, Mannheim, Germany). ELISA kits were used to measure midkine (pg/mL; PeproTech, Hamburg, Germany) and hepcidin-25 (ng/mL, C-ELISA, Intrinsic Lifesciences, LLC, La Jolla, CA, USA), according to the manufacturer's instructions [21].

Serum creatinine was measured using the enzymatic method standardized by isotope dilution mass spectroscopy (Cobas 8000 modular analyzer, Roche Diagnostics). Laboratory investigators were blinded to the sample sources and clinical outcome.

\section{Statistical analysis}

We assessed the discriminative ability and performance of candidate biomarkers to predict study endpoints in a stepwise approach: First, the area under the ROC curve (AUC) based on biomarker concentrations and biomarker/hepcidin-25-ratios measured at ICU admission in conjunction with clinical study endpoints was calculated separately for each marker or ratio (for hepcidin-25, a marker for absence of MAKE and AKI, with reversed orientation indicated as 1-hepcidin-25).

The following variables from the Cleveland kidney risk assessment model were included into the reference logistic regression model [3]: gender, congestive heart failure defined as NYHA (New York Hearth Association classification) class 3 or 4 or left ventricular ejection fraction (LVEF) $<35 \%$, chronic kidney disease defined as preoperative creatinine $>120 \mu \mathrm{mol} / \mathrm{L}$, insulindependent diabetes mellitus, chronic obstructive pulmonary disease, history of previous cardiac surgery, and type of surgery (defined as coronary artery bypass graft [CABG], valve procedure or concomitant procedure). We did not include the variables "emergency surgery" and "use of intra-aortic balloon pump (IABP)" from the original model as all patients underwent elective open-heart surgery and no patient required IABP [3]. The predictive performance of the risk assessment models is reported as AUC for the derived multivariate scores with a 95\% confidence interval (Cl).

Thereafter, we sequentially included each biomarker separately into the reference model to exclude interaction. The model odds ratio (Exp [B]) was calculated to assess the ability of a biomarker to independently predict the study endpoints. The goodness of fit of each logistic regression was assessed using the Hosmer-Lemeshow test.

Reclassification statistics offer additional information not available from the AUC for the quantification of incremental improvements in multivariate model performance following the addition of a candidate biomarker to a reference model [6]. Therefore, improvements in the performance of the reference model following the addition of a urinary biomarker were evaluated by net reclassification improvement (NRI) (reported as categoryfree NRI [cfNRI] to overcome the shortcomings of NRI), and the integrated discrimination improvement (IDI) illustrated as risk assessment plots [22].

To better quantify how accurately the reference and reclassification model would perform with independent data, we adopted a leave-one-out cross-validation. Logarithmic transformations were applied when necessary. SPSS, version 25.0 (IBM Corp., Armonk, NY, USA) and SAS, version 9.4 (SAS Institute Inc., Cary, NC, USA) were used for statistical analysis. $P<0.05$ (twosided) was considered statistically significant.

\section{RESULTS}

\section{Patient characteristics}

Patient baseline characteristics are shown in Table 1. Patients 
Table 1. Perioperative patient characteristics

\begin{tabular}{|c|c|c|c|c|c|c|}
\hline Variable & $\begin{array}{c}\text { No MAKE } \\
N=91\end{array}$ & $\begin{array}{l}\text { MAKE } \\
N=9\end{array}$ & $P^{*}$ & $\begin{array}{l}\text { No AKI } \\
\mathrm{N}=91\end{array}$ & $\begin{array}{c}\text { AKI } \\
N=9\end{array}$ & $P^{\dagger}$ \\
\hline Age (yr) & $67(56-73)$ & $74(70-77)$ & 0.013 & $67(56-73)$ & $74(70-74)$ & 0.015 \\
\hline Sex, female, $N(\%)$ & $30(33.3)$ & $3(33.3)$ & 1.000 & $31(34.1)$ & 2 (22.2) & 0.377 \\
\hline $\begin{array}{l}\text { Insulin-dependent diabetes } \\
\text { mellitus, N (\%) }\end{array}$ & $4(4.4)$ & $0(0)$ & 1.000 & $4(4.4)$ & $0(0)$ & 1.000 \\
\hline Arterial hypertension, N (\%) & $66(72.5)$ & $7(77.8)$ & 0.735 & 65 (71.4) & $8(88.9)$ & 0.439 \\
\hline $\begin{array}{l}\text { Preoperative creatinine }>120 \\
\mu \mathrm{mol} / \mathrm{L}, \mathrm{N}(\%)\end{array}$ & $8(8.8)$ & $4(44.4)$ & 0.011 & $10(11.0)$ & $2(22.2)$ & 0.294 \\
\hline $\begin{array}{l}\text { Preoperative serum creatinine } \\
(\mu \mathrm{mol} / \mathrm{L})\end{array}$ & $86.6(77.8-101.7)$ & $105(77-159)$ & 0.185 & $87(78-105)$ & $88.0(77.0-127.5)$ & 0.558 \\
\hline $\begin{array}{l}\text { Preoperative eGFR mL/min (CKD- } \\
\text { EPI) }\end{array}$ & $72.8(58.8-86.9)$ & $53.6(38.5-85.1)$ & 0.057 & $71.9(57.2-86.9)$ & $57.1(48.3-85.1)$ & 0.226 \\
\hline $\begin{array}{l}\text { Left ventricular dysfunction, } \\
\mathrm{N}(\%)^{\ddagger}\end{array}$ & $15(16.5)$ & $4(44.4)$ & 0.064 & $15(16.5)$ & $4(44.4)$ & 0.064 \\
\hline $\begin{array}{l}\text { Chronic obstructive pulmonary } \\
\text { disease, N (\%) }\end{array}$ & $2(22.2)$ & $9(9.9)$ & 0.257 & $8(8.8)$ & $3(33.3)$ & 0.058 \\
\hline \multicolumn{7}{|l|}{ Procedures } \\
\hline CABG surgery, $N(\%)$ & 17 (18.7) & $2(22.2)$ & 0.679 & 17 (18.7) & $2(22.2)$ & 0.679 \\
\hline Valvular surgery, N (\%) & $46(50.5)$ & $3(33.3)$ & 0.488 & $45(49.5)$ & $4(44.4)$ & 1.000 \\
\hline $\begin{array}{l}\text { CABG and valvular surgery, } \\
\mathrm{N}(\%)\end{array}$ & $21(23.1)$ & $4(44.4)$ & 0.222 & $22(24.2)$ & $3(33.3)$ & 0.687 \\
\hline Redo cardiac surgery, N (\%) & $27(29.7)$ & $1(11.1)$ & 0.438 & $26(28.6)$ & $2(22.2)$ & 1.000 \\
\hline \multicolumn{7}{|c|}{ Biomarker Concentrations 6 hours after commencement of CPB } \\
\hline $\mathrm{NGAL}(\mathrm{ng} / \mathrm{mL})$ & $7.50(0.70-25.40)$ & $69.10(28.95-361.25)$ & 0.010 & $7.50(0.20-25.40)$ & $108.30(28.95-361.25)$ & 0.002 \\
\hline Interleukin-6 (pg/mL) & $6.21(3.54-15.45)$ & 45.59 (20.71-189.99) & 0.001 & $6.21(3.53-15.59)$ & 45.59 (15.03-189.99) & 0.001 \\
\hline Midkine (pg/mL) & 126.50 (96.13-234.63) & $569.50(132.50-4,044.50)$ & 0.011 & 124.75 (94.63-232.75) & $569.50(208.25-4,044.50)$ & 0.001 \\
\hline Hepcidin-25 (ng/mL) & $792.70(254.70-1,565.80)$ & 80.30 (44.40-445.20) & 0.001 & $792.70(254.70-1,565.80)$ & $89.00(56.30-501.65)$ & 0.004 \\
\hline Alpha-1 microglobulin (ng/mL) & $18.40(13.20-31.30)$ & $11.40(6.89-29.30)$ & 0.132 & $18.10(12.10-30.10)$ & $15.90(8.53-53.05)$ & 0.665 \\
\hline \multicolumn{7}{|l|}{ Adverse Outcome } \\
\hline $\begin{array}{l}\text { Postoperative renal } \\
\text { replacement therapy, N (\%) }\end{array}$ & $0(0)$ & $5(55.6)$ & $<0.001$ & $2(2.2)$ & $3(33.3)$ & 0.005 \\
\hline In-hospital mortality, N (\%) & $0(0)$ & $3(33.3)$ & 0.001 & $0(0)$ & $3(33.3)$ & 0.001 \\
\hline MAKE, N (\%) & - & - & - & $2(2.2)$ & $7(77.8)$ & $<0.001$ \\
\hline AKI, N (\%) & $2(2.2)$ & $7(77.8)$ & $<0.001$ & - & - & - \\
\hline
\end{tabular}

Numbers denote median (25th-75th percentile) or $\mathrm{N}(\%)$ where appropriate.

${ }^{\star} P$ for MAKE vs. No-MAKE; ${ }^{\dagger} P$ refers to comparison between AKI and No AKI patients; ${ }^{\ddagger}$ Congestive heart failure defined as NYHA class 3 or 4 or LVEF $<35 \%$.

Abbreviations: AKI, acute kidney injury; CABG, coronary artery bypass graft; CKD-EPI, Chronic Kidney Disease Epidemiology Collaboration equation for estimation of glomerular filtration rate; CPB, cardio-pulmonary bypass; eGFR, estimated glomerular filtration rate; MAKE, major adverse kidney events; NGAL, urine neutrophil gelatinase associated lipocalin; RIFLE, risk injury failure end-stage renal disease classification [18]; NYHA, New York Hearth Association classification; LVEF, left ventricular ejection fraction.

with postoperative MAKE or AKI were older and more likely to have left ventricular dysfunction than patients without MAKE or AKI. Patients with MAKE more frequently had lower baseline estimated glomerular filtration rate (eGFR) than those without
MAKE. Type of surgery and gender were similar in patients with and without MAKE or AKI. AKI was associated with increased risk of acute RRT initiation $(P=0.005)$ and in-hospital mortality $(P=0.001)$. 
Discriminative performance of urinary biomarkers

For MAKE, interleukin- 6 and 1-hepcidin-25 had an AUC of 0.83 (0.68-0.98) and 0.83 (0.72-0.94), respectively. For AKI, NGAL had an AUC of 0.81 (0.67-0.94), interleukin-6 0.82 (0.690.96), and midkine 0.83 (0.69-0.96). Alpha-1 microglobulin was not predictive for either MAKE or AKI (AUC <0.5). The AUC findings for all biomarkers were higher when the biomarker combinations were expressed as $1 /$ hepcidin- 25 ratios. NGAL/ hepcidin-25 ratio was the best performing biomarker for AKI (AUC 0.89 [0.82-0.97]), while interleukin-6/hepcidin-25 demonstrated the highest discriminative performance for MAKE, with an AUC of 0.91 (0.79-1.00). The univariate AUC values are shown in Fig. 2.

Urinary biomarkers as independent predictors of MAKE or AKI We found that NGAL, interleukin-6, midkine, hepcidin-25, and
NGAL/hepcidin-25 were independent predictors of MAKE, while NGAL, interleukin-6, midkine, their hepcidin-25 ratios and hepcidin-25 were independent predictors of AKI using multivariate logistic regression based on the Cleveland Score (Table 2 and Supplemental Data Table S1). Alpha-1 microglobulin and alpha-1 microglobulin/hepcidin-25 were not independently associated with MAKE or AKI.

\section{Estimation of improvement of the Cleveland Score with added urinary biomarker data}

The cross-validated baseline performance characterized by AUC for the Cleveland reference models was 0.84 (0.73-0.96) for MAKE and 0.79 (0.66-0.91) for AKI. The goodness of fit indicated good calibration of the reference kidney risk assessment model for both endpoints (MAKE: Hosmer-Lemeshow, $P=$ 0.864; -2 Log likelihood, 51.33; AKI: Hosmer-Lemeshow, $P=$

A

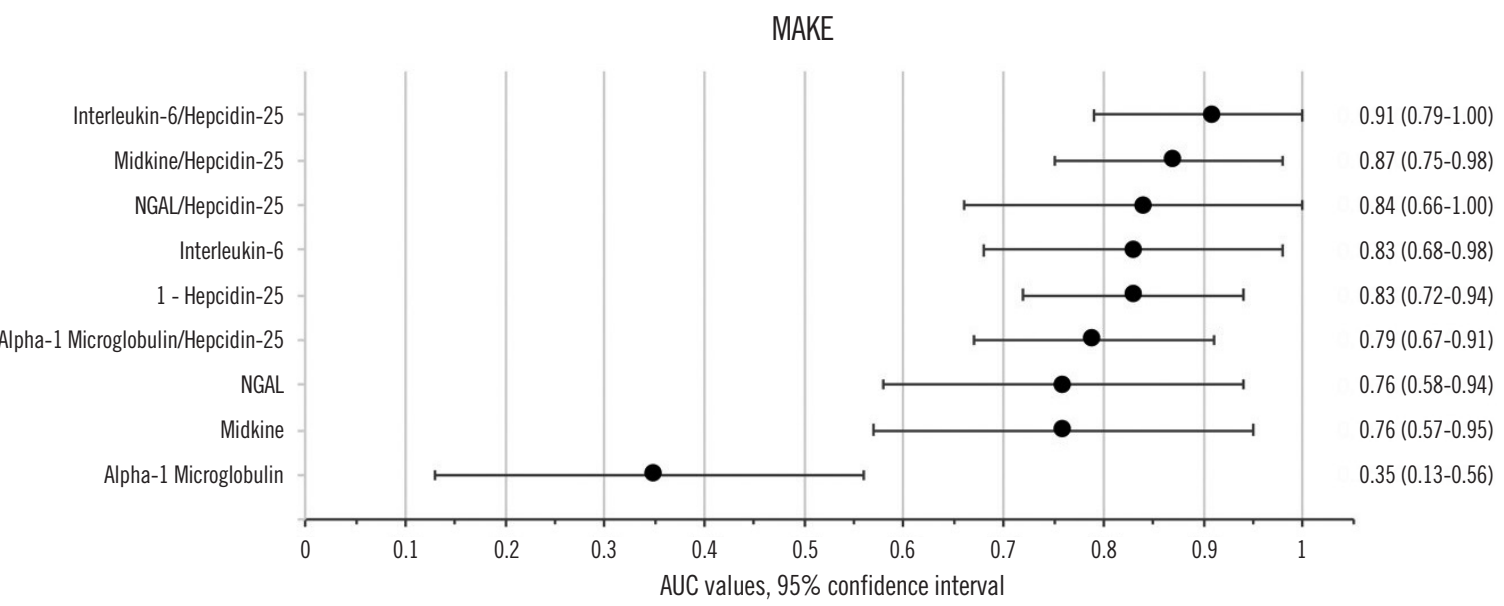

B

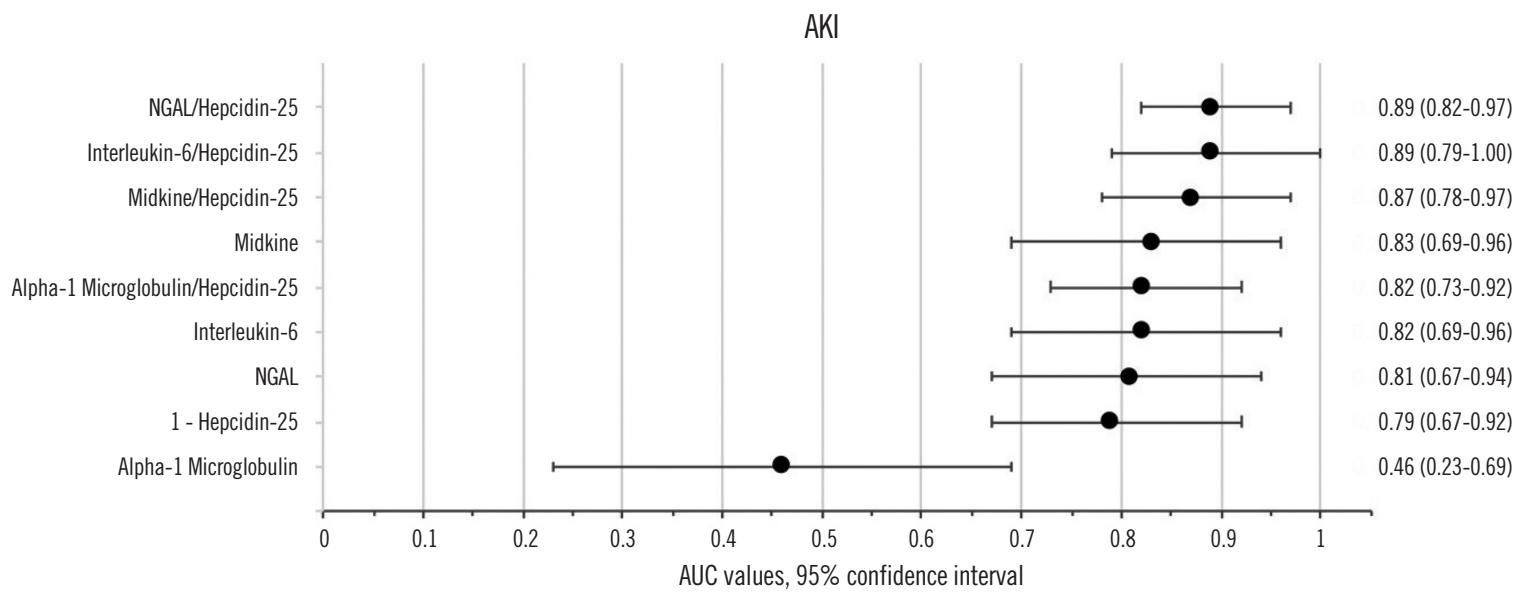

Fig. 2. Ranking of assessed urinary biomarker performance according to the univariate AUC (with 95\% confidence interval bars) at ICU admission for predicting (A) MAKE and (B) AKI.

Abbreviations: NGAL, neutrophil gelatinase-associated lipocalin; ICU, intensive care unit; AUC, area under the ROC curve; MAKE, major adverse kidney events; AKI, acute kidney injury. 
0.968; -2 Log likelihood, 45.28).

The AUC performance of the new model compared with the reference model with $95 \% \mathrm{Cl}$ (orange) is illustrated in Fig. 3. Generally, the addition of urinary biomarkers NGAL, interleukin-6, and midkine, as well as corresponding 1/hepcidin-25 ratios improved the reference model for MAKE and AKI, while alpha-1 microglobulin did not improve model performance (Table 2).

Risk assessment plots illustrating the performance of the reference kidney risk model and the new model are shown in Fig. 4 and Supplemental Data Fig. S1 and S2. Interleukin-6 improved MAKE prediction for events and non-events; however, for AKI this was only true for non-events. In contrast, NGAL improved cfNRI-based AKI prediction of the Cleveland Score for events and non-events; however, for MAKE this was only true for non-events. Only the addition of the NGAL/hepcidin-25 ratio improved the reference model for both MAKE and AKI events and non-events. Midkine and the midkine/hepcidin-25 ratio improved the cfNRI-based prediction of the reference model only for non-events of MAKE and AKI. Finally, IDI, which takes into account the magnitude of changes in predicted risk, showed no improvement for all biomarkers for both endpoints.

\section{DISCUSSION}

This pilot study used established reclassification metrics to assess the incremental value of various urinary biomarkers added

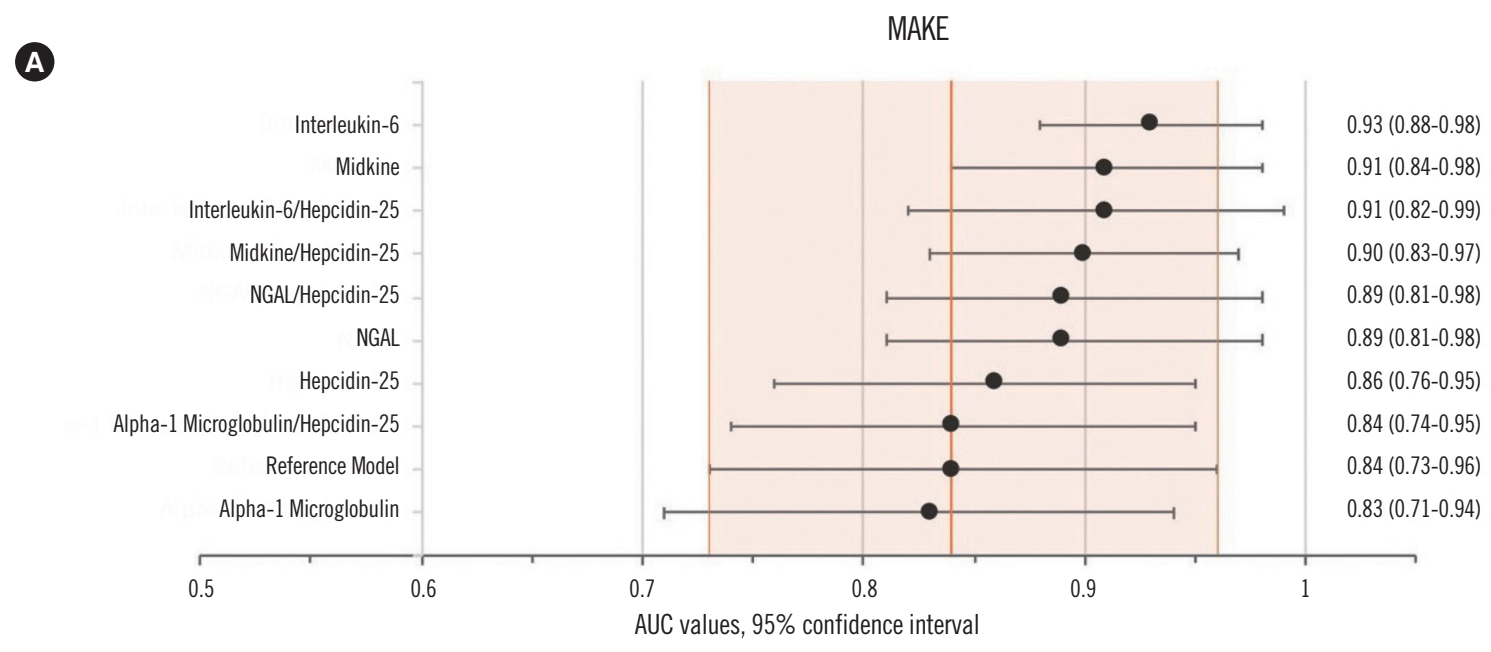

B

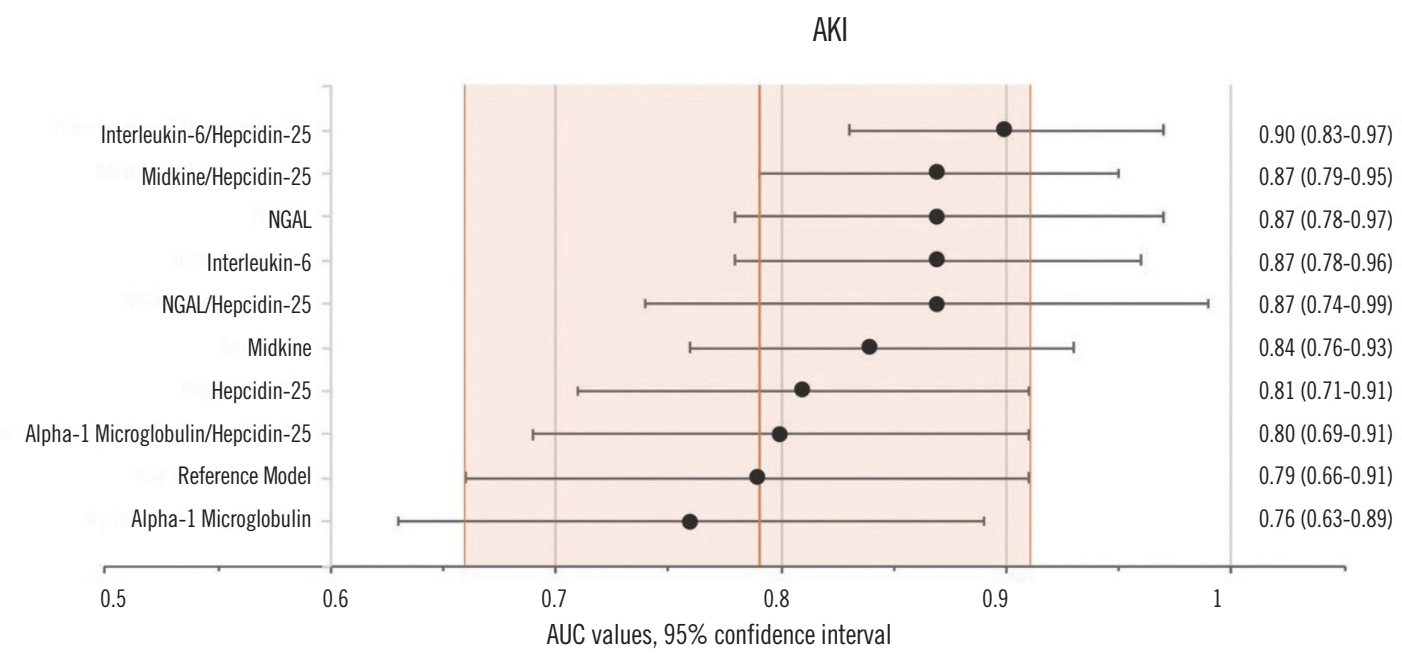

Fig. 3. Ranking of kidney risk prediction model performance to predict (A) MAKE and (B) AKI according to the area under the ROC curve (AUC with 95\% confidence interval [CI] bars) with added urinary kidney injury biomarker at ICU admission (new model) and without (reference model [3], 95\% Cl highlighted orange).

Abbreviations: MAKE, major adverse kidney events; AKI, acute kidney injury; NGAL, neutrophil gelatinase-associated lipocalin; AUC, area under the ROC curve. 
Table 2. Category-free net reclassification improvement (cfNRI) and integrated discrimination improvement (IDI) of selected urinary biomarkers measured at ICU admission

\begin{tabular}{|c|c|c|c|c|c|c|c|c|}
\hline \multirow{3}{*}{$\operatorname{Exp}(B)$} & \multicolumn{4}{|c|}{ MAKE as dependent endpoint } & \multicolumn{4}{|c|}{ AKI as dependent endpoint } \\
\hline & Estimate $(95 \%$ Cl) & $P$ & Estimate $(95 \% \mathrm{Cl})$ & $P$ & Estimate (95\% Cl) & $P$ & Estimate (95\% Cl) & $P$ \\
\hline & \multicolumn{2}{|l|}{ NGAL } & \multicolumn{2}{|c|}{ NGAL/Hepcidin-25 } & \multicolumn{2}{|l|}{ NGAL } & \multicolumn{2}{|c|}{ NGAL/Hepcidin-25 } \\
\hline cfNRI $\left.\right|_{\text {events }}$ & $0.333(-0.283-0.949)$ & 0.289 & $0.556(0.012-1.099)$ & 0.045 & $0.556(0.012-1.099)$ & 0.045 & $0.556(0.012-1.099)$ & 0.045 \\
\hline cfNRI $I_{\text {nonevents }}$ & $0.802(0.680-0.925)$ & $<0.001$ & $0.758(0.624-0.892)$ & $<0.001$ & $0.670(0.518-0.823)$ & $<0.001$ & $0.582(0.415-0.749)$ & $<0.001$ \\
\hline IDI events $_{\text {. }}$ & $0.082(-0.132-0.295)$ & 0.454 & $0.073(-0.144-0.291)$ & 0.509 & $0.162(0.012-0.336)$ & 0.068 & $0.176(-0.001-0.352)$ & 0.051 \\
\hline$|D|_{\text {nonevents }}$ & $-0.006(-0.027-0.014)$ & 0.550 & $-0.006(-0.028-0.017)$ & 0.625 & $0.015(0.045-0.016)$ & 0.342 & $-0.016(-0.047-0.016)$ & 0.333 \\
\hline IDI & $0.075(-0.139-0.290)$ & 0.491 & $0.068(-0.151-0.286)$ & 0.543 & $0.147(0.029-0.324)$ & 0.102 & $0.160(-0.019-0.339)$ & 0.081 \\
\hline$A U C_{\text {difference }}$ & $0.051(-0.044-0.147)$ & 0.291 & $0.049(-0.078-0.175)$ & 0.449 & $0.085(-0.049-0.220)$ & 0.212 & $0.079(-0.088-0.246)$ & 0.351 \\
\hline cfNRI $I_{\text {nonevents }}$ & $0.733(0.593-0.874)$ & $<0.001$ & $0.556(0.384-0.727)$ & $<0.001$ & $0.533(0.359-0.708)$ & $<0.001$ & $0.422(0.235-0.610)$ & $<0.001$ \\
\hline cfNRI & $1.289(0.728-1.850)$ & $<0.001$ & $1.111(0.541-1.681)$ & $<0.001$ & $0.867(0.226-1.507)$ & 0.008 & $0.756(0.112-1.399)$ & 0.021 \\
\hline$|D|_{\text {events }}$ & $0.122(-0.121-0.364)$ & 0.325 & $0.097(-0.144-0.339)$ & 0.429 & $0.138(-0.067-0.343)$ & 0.186 & $0.173(-0.028-0.373)$ & 0.091 \\
\hline $\mathrm{IDI}_{\text {nonevents }}$ & $-0.009(-0.038-0.021)$ & 0.567 & $-0.011(-0.035-0.013)$ & 0.382 & $-0.009(-0.033-0.015)$ & 0.463 & $-0.013(-0.039-0.013)$ & 0.331 \\
\hline IDI & $0.113(-0.131-0.357)$ & 0.363 & $0.087(-0.156-0.329)$ & 0.484 & $0.129(-0.077-0.335)$ & 0.219 & $0.160(-0.043-0.362)$ & 0.122 \\
\hline \multirow[t]{2}{*}{$A U C_{\text {difference }}$} & $0.089(-0.006-0.183)$ & 0.065 & $0.064(-0.068-0.197)$ & 0.342 & $0.085(-0.007-0.177)$ & 0.069 & $0.114(0.007-0.220)$ & 0.036 \\
\hline & \multicolumn{2}{|l|}{ Midkine } & \multicolumn{2}{|c|}{ Midkine/Hepcidin-25 } & \multicolumn{2}{|l|}{ Midkine } & \multicolumn{2}{|c|}{ Midkine/Hepcidin-25 } \\
\hline $\operatorname{Exp}(B)$ & $1.001(1.000-1.001)$ & 0.031 & $1.115(0.990-1.256)$ & 0.072 & $1.001(1.000-1.001)$ & 0.020 & $1.163(1.001-1.350)$ & 0.048 \\
\hline \multirow[t]{2}{*}{$A U C_{\text {difference }}$} & $0.065(-0.025-0.156)$ & 0.157 & $0.056(-0.061-0.173)$ & 0.352 & $0.058(-0.045-0.161)$ & 0.269 & $0.081(-0.023-0.186)$ & 0.128 \\
\hline & \multicolumn{2}{|c|}{ Alpha-1 Microglobulin } & \multicolumn{2}{|c|}{ Alpha-1 Microglobulin/Hepcidin-25 } & \multicolumn{2}{|c|}{ Alpha-1 Microglobulin } & \multicolumn{2}{|c|}{ Alpha-1 Microglobulin/Hepcidin-25 } \\
\hline Exp (B) & $0.993(0.972-1.014)$ & 0.493 & $1.676(0.443-6.335)$ & 0.447 & $0.979(0.933-1.027)$ & 0.383 & $0.996(0.212-4.677)$ & 0.996 \\
\hline$\left.c f N R\right|_{\text {events }}$ & $0.333(-0.283-0.949)$ & 0.289 & $-0.111(-0.760-0.538)$ & 0.737 & $-1.000(-1.000--1.000)$ & 0.317 & $0.556(0.012-1.099)$ & 0.045 \\
\hline cfNRI $I_{\text {nonevents }}$ & $-0.209(-0.410-0.008)$ & 0.042 & $0.055(-0.150-0.260)$ & 0.600 & $0.253(0.054-0.452)$ & 0.013 & $0.187(-0.015-0.389)$ & 0.070 \\
\hline cfNRI & $0.125(-0.523-0.773)$ & 0.706 & $-0.056(-0.737-0.625)$ & 0.872 & $-0.747(-0.956--0.549)$ & $<0.001$ & $0.742(0.163-1.322)$ & 0.012 \\
\hline$|D|_{\text {events }}$ & $-0.008(-0.053-0.036)$ & 0.710 & $-0.025(-0.071-0.021)$ & 0.286 & $-0.030(-0.049--0.010)$ & 0.003 & $0.009(-0.049-0.067)$ & 0.766 \\
\hline$|D|_{\text {nonevents }}$ & $0.000(-0.007-0.007)$ & 0.962 & $0.004(-0.004-0.013)$ & 0.284 & $0.003(-0.001-0.006)$ & 0.135 & $0.003(-0.015-0.020)$ & 0.779 \\
\hline IDI & $-0.009(-0.053-0.036)$ & 0.708 & $-0.021(-0.068-0.026)$ & 0.387 & $-0.027(-0.047--0.007)$ & 0.007 & $0.011(-0.049-0.072)$ & 0.715 \\
\hline$A U C_{\text {difference }}$ & $-0.016(-0.061-0.029)$ & 0.488 & $0.001(-0.016-0.018)$ & 0.889 & $-0.031(-0.049--0.012)$ & 0.001 & $0.016(-0.043-0.074)$ & 0.595 \\
\hline
\end{tabular}

Goodness of fit for reference models: MAKE: Hosmer-Lemeshow $P=0.864,-2$ Log likelihood 51.33, Nagelkerke R² 0.195, AUC 0.84; AKI: Hosmer-Lemeshow $P=0.968$, -2 Log likelihood 45.28, Nagelkerke $R^{2} 0.311$, AUC 0.79 .

Abbreviations: AKI, acute kidney injury; MAKE, major adverse kidney events; AUC, area under the curve; Cl, confidence interval; cfNRI, category-free net reclassification improvement; $\operatorname{Exp}(B)$, Logit coefficient expressed as odds ratio for one performance unit; NGAL, neutrophil gelatinase-associated lipocalin; IDI, integrated discrimination improvement. 


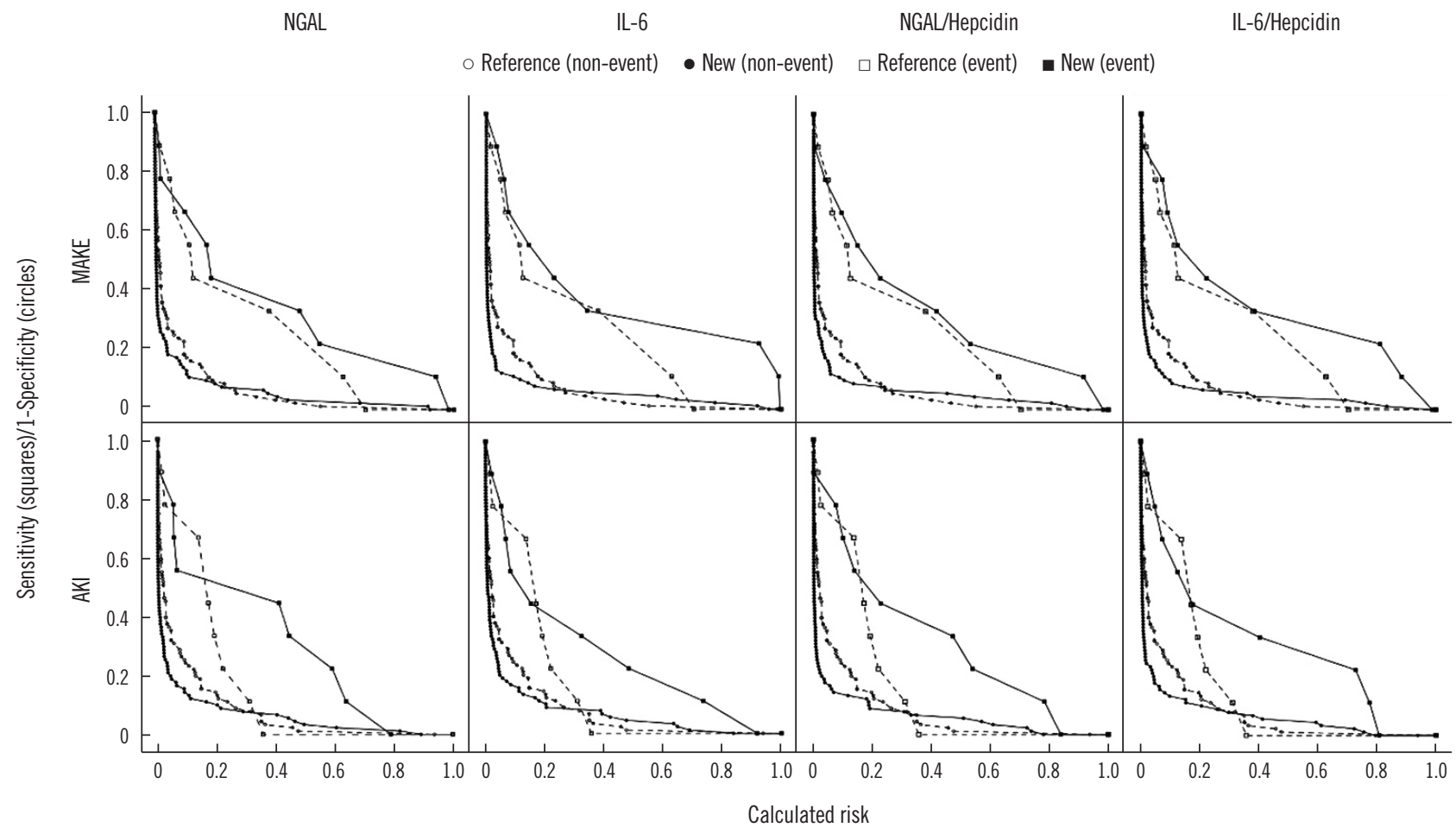

Fig. 4. Risk assessment plots showing the changes in model performance. Compared with AUC graphs, risk assessment plots illustrate infor-

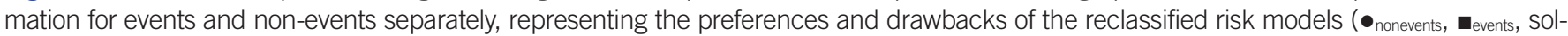
id lines) calculated by the addition of urinary biomarker concentrations (NGAL, interleukin-6, NGAL/hepcidin-25, interleukin-6/hepcidin-25) to the reference model ( $O_{\text {nonevents, }} \square$ events, dashed lines). $\square$ represent model sensitivity ( $Y$-axis) versus the calculated risk ( $X$-axis) for those with the event. $\bullet$ represent 1 -specificity ( $Y$-axis) versus the calculated risk ( $X$-axis) for those without an event (endpoints MAKE, AKI).

Abbreviations: AUC, area under the ROC curve; MAKE, major adverse kidney events; AKI, acute kidney injury; NGAL, neutrophil gelatinase-associated lipocalin; IL-6, interleukin-6.

to the Cleveland Score to predict MAKE and AKI after cardiac surgery [3]. We found that urinary NGAL, interleukin-6, and their 1/hepcidin-25-ratios are independent predictors that improve cfNRI-based MAKE- and AKI-prediction when added to the Cleveland Score. Notably, biomarker-related improvement was particularly dependent on correctly reclassifying patients without the event of interest.

Several statistical proposals have been made to assess the additional benefit of predictive biomarkers. Comparative metrics (AUC, cfNRI, IDI) are differently sensitive to detecting small improvements. Specifically, we found that high cfNRI $I_{\text {nonevent }}$ values indicate the ability of a biomarker to correctly decrease risk estimates for non-events. Thus, they are useful for ruling out adverse kidney events [23]. In contrast, IDI and cfNRI levent are presumably confounded by low event numbers for MAKE and AKI [24].

As no single kidney biomarker will meet every requirement regarding the underlying pathophysiological mechanisms of AKI, we assessed the predictive performance of several urinary biomarkers representing different direct or indirect components of tubular stress or damage $[13,25]$ or potential renal protective mechanisms $[15,16]$. We found increased postoperative urinary hepcidin-25 concentrations in non-AKI patients, whereas the concentrations remained low in patients with subsequent $A K I$, in line with previous studies $[15,16,26]$. We hypothesize that the improved predictive ability of NGAL and interleukin-6 expressed as 1/hepcidin-25 ratio vs NGAL and interleukin-6 alone may be explained by the role of hepcidin- 25 in the metabolism of labile-iron compounds in the presence and absence of AKI [15, 26-28]. During cardiac surgery, unbound labile ironrelated injury maintained by oxido-inflammatory stress may be of specific pathophysiological importance [7]. The involvement and contribution of interleukin- 6 to inflammatory responsepathways have also been implicated in the pathogenesis of ischemic AKI [29]. We believe that interleukin-6, a pro-inflammatory cytokine detected in urine, in particular, may serve as an indicator of tubular oxido-inflammation in cardiac surgery associated kidney injury. We were also able to extend previous findings on midkine as a potentially valuable predictor of MAKE and 
AKI [14]. Finally, the weak discriminatory ability of alpha-1 microglobulin for MAKE and AKI after cardiac surgery may be related to other predominant pathogenic tubular stress factors being effective in cardiac surgery compared with critically ill patients for whom alpha-1 microglobulin was previously found to be of value [12].

Perioperative risk assessment provides an opportunity for early diagnosis of adverse kidney events and early implementation of interventional strategies [30, 31]. Our finding regarding improved biomarker risk assessment after cardiac surgery carries the potential of timely implementation of AKI care bundles, triggering diagnostic and therapeutic modifications [32], which are associated with favorable outcomes [30]. Importantly, given the pronounced improvement in non-event prediction, our findings may facilitate withholding unnecessary diagnostic or therapeutic measures. Considering the performance and immediate availability of the assessed biomarkers in clinical practice, NGAL and interleukin-6, both measurable using clinical laboratory platforms, appear to be the most promising candidates for implementation in kidney risk assessment at the bedside. Finally, our findings on alpha-1 microglobulin are in line with previous findings and do not support further investigation of this biomarker in the cardiac surgery setting [33].

Our study has several strengths and limitations. We investigated typical patients at risk of adverse outcome in a relatively homogenous and well-defined patient cohort after cardiac surgery. Well-calibrated statistical and clinical risk assessment models enabled us to identify specific predictive patterns such as biomarkers improving prediction of non-events. In patient cohorts with only a few MAKE or AKI events, large sample sizes are necessary to derive reliable performance estimates, particularly for the estimation of classification errors. Thus, the limited sample size of this study is clearly a limitation. However, the apparent improvement in the accuracy of prediction models with small sample sizes may be potentially confounded by model overfitting. Hence, we utilized cross-validated estimates. As expected, model accuracy (AUC) was decreased, and the crossvalidated measures were more conservative in detecting model improvement [34]. Weaker performance in reclassifying "events" may also reflect the limitations of the markers themselves or may be, at least partly, explained by "imperfect" clinical endpoints, which commonly depend on changes in serum creatinine, a poor reference standard [35]. Finally, the present study does not preclude the conclusion that no other kidney biomarkers may improve AKI risk prediction, as previously shown [36].
Early recognition of AKI or subclinical AKI using NGAL or interleukin- 6 analysis at the bedside may help guide early riskstratified interventions, such as implementation of the Kidney Disease Improving Global Outcomes (KDIGO) care bundles, and may also prevent unnecessary care for event-free patients [24, 30]. Future studies should clarify whether such clinical kidney risk assessment and interventions guided by readily available biomarkers can improve outcomes.

In summary, biomarkers from multiple, biologically linked pathways related to iron metabolism and/or inflammation are associated with the risk of adverse kidney related outcomes [37, 38]. In this pilot study, we found urinary NGAL and interleukin-6 to be the most promising candidates for further clinical implementation research. The combination of biomarkers with hepcidin-25 may further improve diagnostic discrimination. Further research into the reno-protective abilities of hepcidin- 25 should be conducted.

\section{Acknowledgements}

We thank Argid and Raimund Rutenberg for their excellent research assistance.

\section{Author Contributions}

$\mathrm{CA}, \mathrm{MH}, \mathrm{AA}, \mathrm{SK}, \mathrm{RB}, \mathrm{RBD}, \mathrm{AHF}$ made substantial contributions to conception, design and analysis of the study. $\mathrm{MH}$ and $\mathrm{AHF}$ performed acquisition of patient data. MW, SW performed laboratory testing. SK, CA performed statistical analysis. All authors participated in drafting and/or revising the paper and provided important intellectual contributions. All authors accepted their responsibility for the entire content of the manuscript, gave final approval of the submitted version and any revised versions submitted prior to acceptance.

\section{Conflicts of Interest}

CA has received honoraria speaking for Siemens Healthineers. $\mathrm{MH}$ has received honoraria speaking for Abbott Diagnostics, Alere, Biosite Inc., and Siemens Healthineers, AA has received honoraria speaking for Abbott Diagnostics. All companies are involved in the development and marketing of renal biomarkers.

\section{Research Funding}

This study was supported by grants from the German Heart 
Foundation (Deutsche Stiftung für Herzforschung) and from the Else Kröner-Fresenius-Stiftung, Germany.

\section{ORCID}

\section{Christian Albert https://orcid.org/0000-0002-6956-9962}

Michael Haase https://orcid.org/0000-0001-8212-7416

Annemarie Albert https://orcid.org/0000-0002-5611-1506

Siegfried Kropf https://orcid.org/0000-0002-5197-3481

Rinaldo Bellomo https://orcid.org/0000-0002-1650-8939

Sabine Westphal https://orcid.org/0000-0002-5846-3345

Rüdiger Christian Braun-Dullaeus https://orcid.org/0000-00033888-6532

Anja Haase-Fielitz https://orcid.org/0000-0001-6881-2249

${ }^{\dagger}$ Mark Westerman, deceased, no ORCID available.

\section{REFERENCES}

1. Haase-Fielitz A, Haase M, Bellomo R, Calzavacca P, Spura A, Baraki H, et al. Perioperative hemodynamic instability and fluid overload are associated with increasing acute kidney injury severity and worse outcome after cardiac surgery. Blood Purif 2017;43:298-308.

2. Kellum JA, Lameire N, Aspelin P, Barsoum RS, Burdmann EA, Goldstein SL, et al. Kidney disease: improving global outcomes (KDIGO) acute kidney injury work group. KDIGO clinical practice guideline for acute kidney injury. Kidney Int Suppl 2012;2:1-138.

3. Thakar CV, Arrigain S, Worley S, Yared JP, Paganini EP. A clinical score to predict acute renal failure after cardiac surgery. J Am Soc Nephrol 2005; 16:162-8.

4. Vives M, Monedero P, Perez-Valdivieso JR, Garcia-Fernandez N, Lavilla $\mathrm{J}$, Herreros J, et al. External validation and comparison of three scores to predict renal replacement therapy after cardiac surgery: a multicenter cohort. Int Artif Organs 2011;34:329-38.

5. McCullough PA, Shaw AD, Haase M, Bouchard J, Waikar SS, Siew ED, et al. Diagnosis of acute kidney injury using functional and injury biomarkers: workgroup statements from the tenth Acute Dialysis Quality Initiative Consensus Conference. Contrib Nephrol 2013;182:13-29.

6. Pencina MJ, D'Agostino RB Sr, D'Agostino RB Jr, Vasan RS. Evaluating the added predictive ability of a new marker: from area under the ROC curve to reclassification and beyond. Stat Med 2008;27:157-72.

7. Haase M, Bellomo R, Haase-Fielitz A. Novel biomarkers, oxidative stress, and the role of labile iron toxicity in cardiopulmonary bypass-associated acute kidney injury. J Am Coll Cardiol 2010;55:2024-33.

8. Renhua L, Miaolin C, Junlin W, Qingwei W, Xiaoping X, Huili D, et al. The level of the biomarkers at the time of nephrology consultation might predict the prognosis of acute kidney injury in hospitalized patients. Blood Purif 2014;38:89-95.

9. Gueret G, Lion F, Guriec N, Arvieux J, Dovergne A, Guennegan C, et al. Acute renal dysfunction after cardiac surgery with cardiopulmonary bypass is associated with plasmatic IL6 increase. Cytokine 2009;45:92-8.

10. Haase M, Bellomo R, Devarajan P, Schlattmann P, Haase-Fielitz A, the NGAL Meta-analysis Investigator Group. Accuracy of neutrophil gelatinase-associated lipocalin (NGAL) in diagnosis and prognosis in acute kidney injury: a systemic review and meta-analysis. Am J Kidney Dis
2009;54:1012-24

11. Nemeth $E$ and Ganz T. The role of hepcidin in iron metabolism. Acta Haematol 2009;122:78-86.

12. Herget-Rosenthal S, Poppen D, Hüsing J, Marggraf G, Pietruck F, Jakob HG, et al. Prognostic value of tubular proteinuria and enzymuria in nonoliguric acute tubular necrosis. Clin Chem 2004;50:552-8.

13. Sato W, Kadomatsu K, Yuzawa Y, Muramatsu H, Hotta N, Matsuo S, et al. Midkine is involved in neutrophil infiltration into the tubulointerstitium in ischemic renal injury. J Immunol 2001;167:3463-9.

14. Hayashi H, Sato W, Kosugi T, Nishimura K, Sugiyama D, Asano N, et al. Efficacy of urinary midkine as a biomarker in patients with acute kidney injury. Clin Exp Nephrol 2017;21:597-607.

15. Ho J, Reslerova M, Gali B, Gao A, Bestland J, Rush DN, et al. Urinary hepcidin-25 and risk of acute kidney injury following cardiopulmonary bypass. Clin J Am Soc Nephrol 2011;6:2340-6.

16. Mårtensson J, Glassford NJ, Jones S, Eastwood GM, Young H, Peck L, et al. Urinary neutrophil gelatinase-associated lipocalin to hepcidin ratio as a biomarker of acute kidney injury in intensive care unit patients. Minerva Anestesiol 2015;81:1192-200.

17. Haase M, Haase-Fielitz A, Plass M, Kuppe H, Hetzer R, Hannon C, et al. Prophylactic perioperative sodium bicarbonate to prevent acute kidney injury following open heart surgery: a multicenter double-blinded randomized controlled trial. PLOS Med 2013;10:e1001426.

18. Bellomo R, Ronco C, Kellum JA, Mehta RL, Palevsky P; Acute Dialysis Quality Initiative workgroup. Acute renal failure-definition, outcome measures, animal models, fluid therapy and information technology needs: the Second International Consensus Conference of the Acute Dialysis Quality Initiative (ADQI) Group. Crit Care 2004;8:R204-12.

19. Englberger L, Suri RM, Li Z, Casey ET, Daly RC, Dearani JA, et al. Clinical accuracy of RIFLE and Acute Kidney Injury Network (AKIN) criteria for acute kidney injury in patients undergoing cardiac surgery. Crit Care 2011;15:R16.

20. Xiong J, Tang X, Hu Z, Nie L, Wang Y, Zhao J. The RIFLE versus AKIN classification for incidence and mortality of acute kidney injury in critical ill patients: a meta-analysis. Sci Rep 2015;5:1791717917.

21. Ganz T, Olbina G, Girelli D, Nemeth E, Westerman M. Immunoassay for human serum hepcidin. Blood 2008;112:4292-7.

22. Pencina MJ, D'Agostino RB Sr, Steyerberg EW. Extensions of net reclassification improvement calculations to measure usefulness of new biomarkers. Stat Med 2011;30:11-21.

23. Leening MJG, Vedder MM, Witteman JC, Pencina MJ, Steyerberg EW. Net reclassification improvement: computation, interpretation, and controversies: a literature review and clinician's guide. Ann Intern Med 2014;160:122-31.

24. Albert C, Albert A, Bellomo R, Kropf S, Devarajan P, Westphal S, et al. Urinary neutrophil gelatinase-associated lipocalin-guided risk assessment for major adverse kidney events after open-heart surgery. Biomark Med 2018;12:975-85.

25. Kim SY, Jeong TD, Lee W, Chun S, Sunwoo S, Kim SB, et al. Plasma neutrophil gelatinase-associated lipocalin as a marker of tubular damage in diabetic nephropathy. Ann Lab Med 2018;38:524-9.

26. Prowle JR, Ostland V, Calzavacca P, Licari E, Ligabo EV, Echeverri JE, et al. Greater increase in urinary hepcidin predicts protection from acute kidney injury after cardiopulmonary bypass. Nephrol Dial Transplant 2012;27:595-602.

27. Mori K, Lee HT, Rapoport D, Drexler IR, Foster K, Yang J, et al. Endocytic delivery of lipocalin-siderophore-iron complex rescues the kidney from ischemia-reperfusion injury. J Clin Invest 2005;115:610-21.

28. van Swelm RPL, Wetzels JF, Verweij VG, Laarakkers CM, Pertijs JC, van der Wijst J, et al. Renal handling of circulating and renal-synthesized 
Albert C, et al.

Urinary biomarkers may complement the Cleveland Score

ANNALS OF

LABORATORY MEDICINE

hepcidin and its protective effects against hemoglobin-mediated kidney injury. J Am Soc Nephrol 2016;27:2720-32.

29. Burne-Taney MJ, Kofler J, Yokota N, Weisfeldt M, Traystman RJ, Rabb H. Acute renal failure after whole body ischemia is characterized by inflammation and T cell-mediated injury. Am J Physiol Renal Physiol 2003;285:F87-94.

30. Meersch M, Schmidt C, Hoffmeier A, Van Aken H, Wempe C, Gerss J, et al. Prevention of cardiac surgery-associated AKI by implementing the KDIGO guidelines in high risk patients identified by biomarkers: the PrevAKI randomized controlled trial. Intensive Care Med 2017;43: 1551-61.

31. de Geus HR, Ronco C, Haase M, Jacob L, Lewington A, Vincent JL. The cardiac surgery-associated neutrophil gelatinase-associated lipocalin (CSA-NGAL) score: a potential tool to monitor acute tubular damage. J Thorac Cardiovasc Surg 2016;151:1476-81.

32. Haase M, Kribben A, Zidek W, Floege J, Albert C, Isermann B, et al. Electronic alerts for acute kidney injury. Dtsch Arztebl Int 2017;114:18.

33. Ho J, Lucy M, Krokhin O, Hayglass K, Pascoe E, Darroch G, et al. Mass spectrometry-based proteomic analysis of urine in acute kidney injury following cardiopulmonary bypass: a nested case-control study. Am J Kidney Dis 2009;53:584-95.

34. Airola A, Pahikkala T, Waegeman W, De Baets B, Salakoski T. An experimental comparison of cross-validation techniques for estimating the area under the ROC curve. Comput Stat Data Anal 2011;55:1828-44.

35. Waikar SS, Betensky RA, Emerson SC, Bonventre JV. Imperfect gold standards for kidney injury biomarker evaluation. J Am Soc Nephrol 2012;23:13-21.

36. Kim H, Hur M, Lee S, Marino R, Magrini L, Cardelli P, et al. Proenkephalin, neutrophil gelatinase-associated lipocalin, and estimated glomerular filtration rates in patients with sepsis. Ann Lab Med 2017;37:3889797.

37. Haase M, Devarajan P, Haase-Fielitz A, Bellomo R, Cruz DN, Wagener G, et al. The outcome of neutrophil gelatinase-associated lipocalin-positive subclinical acute kidney injury: a multicenter pooled analysis of prospective studies. J Am Coll Cardiol 2011;57:1752-61.

38. Albert C, Albert A, Kube J, Bellomo R, Wettersten N, Kuppe H, et al. Urinary biomarkers may provide prognostic information for subclinical acute kidney injury after cardiac surgery. J Thorac Cardiovasc Surg 2018;155:2441-2452.e13. 
Supplemental Data Table S1. (Continuation of main manuscript Table 2): Category-free net reclassification improvement (cfNRI) and integrated discrimination improvement (IDI) of urinary hepcidin-25 measured at intensive care unit admission (data corresponding to risk assessment plots in Supplemental Data Fig. S2)

\begin{tabular}{|c|c|c|c|c|c|}
\hline \multirow{2}{*}{ MAKE } & Estimate $(95 \% \mathrm{Cl})$ & $P$ & \multirow{2}{*}{ AKI } & Estimate (95\% Cl) & $P$ \\
\hline & \multicolumn{2}{|c|}{ Hepcidin-25 } & & \multicolumn{2}{|c|}{ Hepcidin-25 } \\
\hline $\operatorname{Exp}(B)$ & $0.996(0.992-1.000)$ & 0.049 & $\operatorname{Exp}(B)$ & $0.996(0.992-1.000)$ & 0.030 \\
\hline cfNRI $\left.\right|_{\text {events }}$ & $0.111(-0.538-0.760)$ & 0.737 & $\mathrm{cfNRI}_{\text {events }}$ & $0.111(-0.538-0.760)$ & 0.737 \\
\hline cfNRI $I_{\text {nonevents }}$ & $-0.121(-0.325-0.083)$ & 0.245 & cfNRI $\left.\right|_{\text {nonevents }}$ & $-0.011(-0.216-0.195)$ & 0.917 \\
\hline cfNRI & $-0.010(-0.690-0.671)$ & 0.978 & cfNRI & $0.100(-0.581-0.781)$ & 0.773 \\
\hline$|D|_{\text {events }}$ & $-0.032(-0.124-0.059)$ & 0.491 & $|D|_{\text {events }}$ & $0.013(-0.046-0.072)$ & 0.667 \\
\hline$|D|_{\text {nonevents }}$ & $0.003(-0.009-0.014)$ & 0.653 & $\left.I D\right|_{\text {nonevents }}$ & $0.001(-0.015-0.016)$ & 0.949 \\
\hline IDI & $-0.030(-0.122-0.063)$ & 0.53 & IDI & $0.013(-0.047-0.074)$ & 0.665 \\
\hline
\end{tabular}

Goodness of fit for reference models: MAKE: Hosmer-Lemeshow $P=0.864,-2$ Log likelihood 51.33, Nagelkerke R2 0.195, AUC 0.84; AKI: Hosmer-Lemeshow $P=0.968$, -2 Log likelihood 45.28, Nagelkerke R2 0.311, AUC 0.79.

Abbreviations: MAKE, major adverse kidney events; AKI, acute kidney injury; IDI, integrated discrimination improvement; cfNRI, category-free net reclassification improvement. 


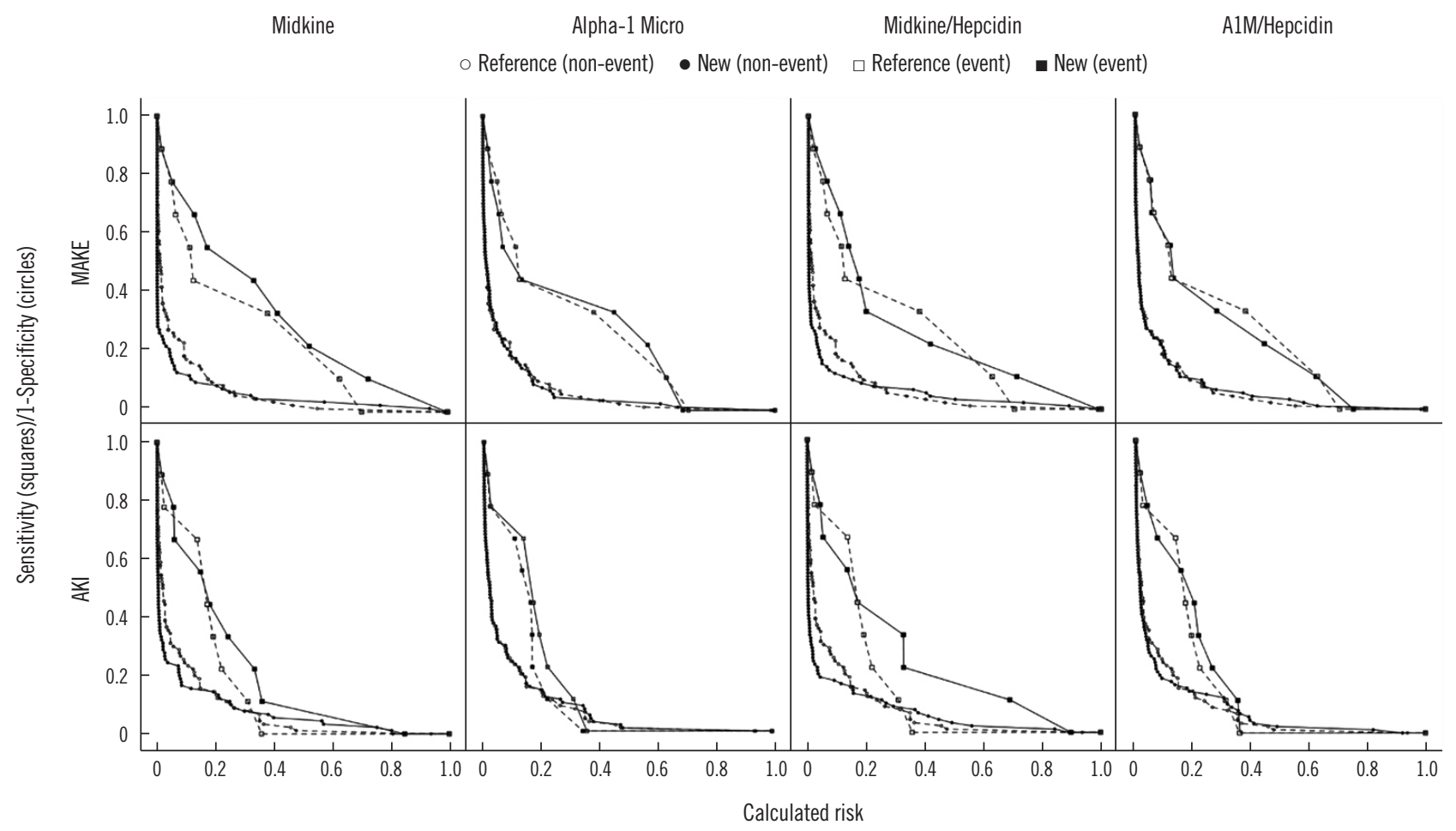

Supplemental Data Fig. S1. Risk-assessment plots for midkine, alpha-1 microglobulin (A1M), midkine/hepcidin-25, A1M/hepcidin-25

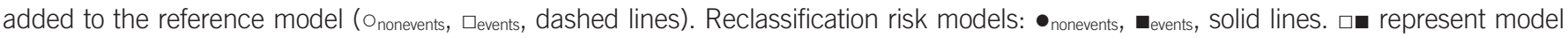
sensitivity $(Y$-axis) versus the calculated risk ( $X$-axis) for those with the event. $\bullet \bullet$ represent 1 -specificity $(Y$-axis) versus the calculated risk ( $X$-axis) for those without an event (endpoints MAKE, AKI).

Abbreviations: MAKE, major adverse kidney events; AKI, acute kidney injury; A1M/Alpha-1 Micro, alpha-1 microglobulin. 

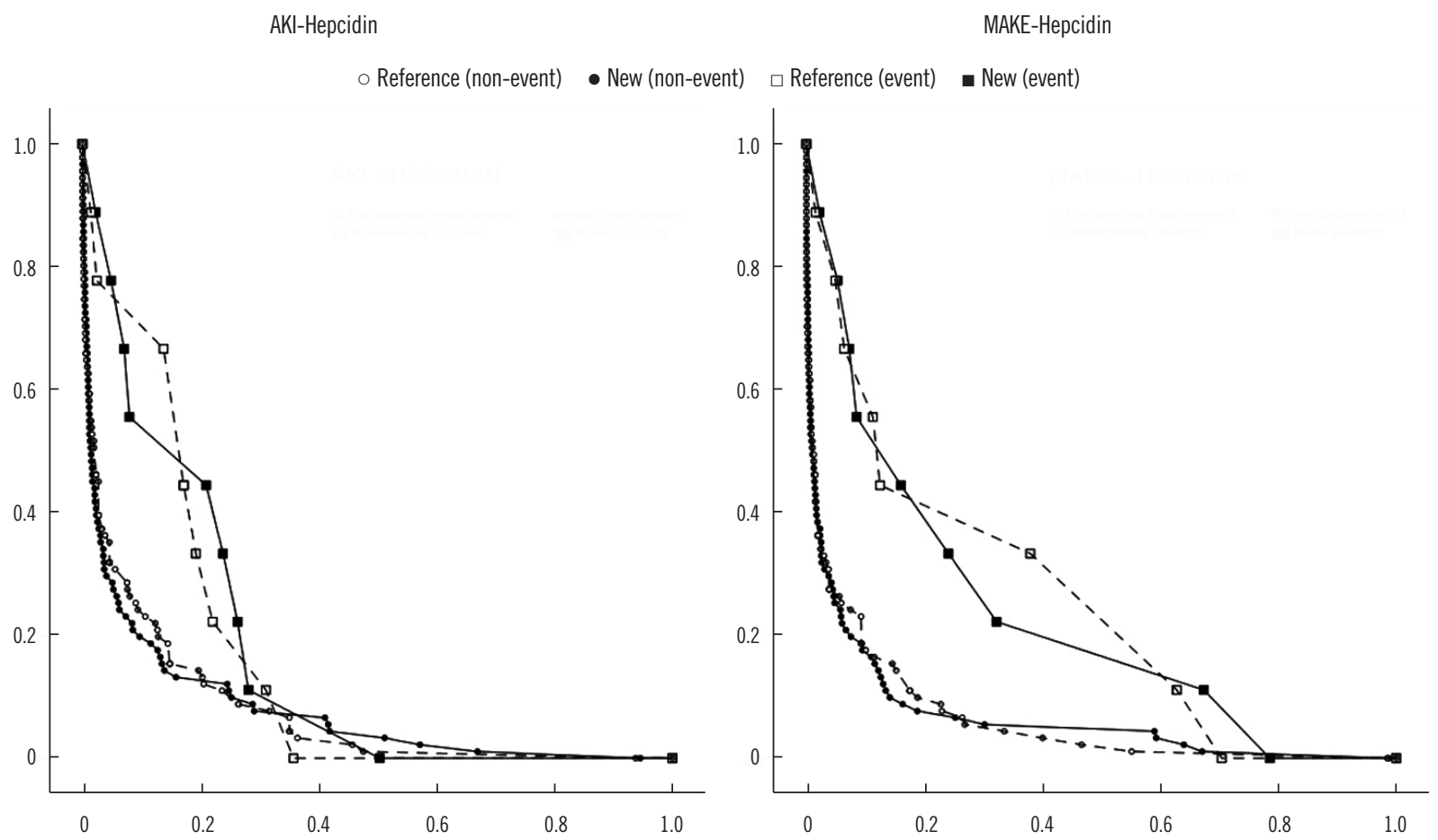

Supplemental Data Fig. S2. Risk-assessment plots for hepcidin added to the reference model (Ononevents, 口events, dashed lines); Reclassifica-

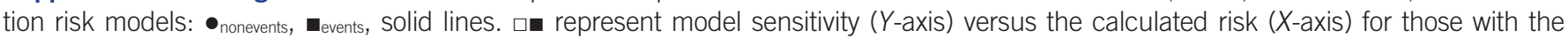
event. $\bullet$ represent 1 -specificity ( $Y$-axis) versus the calculated risk ( $X$-axis) for those without an event (endpoints MAKE, AKI).

Abbreviations: MAKE, major adverse kidney events; AKI, acute kidney injury. 